\title{
Drinking and Obesity: Alcoholic Liver Disease/ Nonalcoholic Fatty Liver Disease Interactions
}

\author{
Fredrik Åberg, MD, $\mathrm{PhD}^{1,2}$ Martti Färkkilä3 \\ 1 Transplantation and Liver Surgery Clinic, Helsinki University \\ Hospital, Helsinki, Finland \\ 2 The Transplant Institute, Sahlgrenska University Hospital, \\ Gothenburg, Sweden \\ ${ }^{3}$ Clinic of Gastroenterology, Helsinki University Hospital, Helsinki, Finland \\ Semin Liver Dis 2020;40:154-162.
}

\begin{abstract}
Address for correspondence Fredrik Åberg, MD, PhD, Transplantation and Liver Surgery Clinic, Helsinki University Hospital, PB 372, Helsinki 00029, Finland (e-mail: Fredrik.Aberg@helsinki.fi).
\end{abstract}

\begin{abstract}
Alcohol and obesity are the main risk factors for alcoholic liver disease and nonalcoholic fatty liver disease (NAFLD), respectively, and they frequently coexist. There are considerable synergistic interaction effects between hazardous alcohol use and obesity-associated metabolic abnormalities in the development and progression of fatty liver disease. Intermittent binge-drinking has been shown to promote steatohepatitis from obesityrelated steatosis, and binge-drinking is associated with progression to cirrhosis even when average alcohol intake is within the currently used criteria for a NAFLD diagnosis. Recent

Keywords

- nonalcoholic fatty liver disease

- alcohol

- fatty liver

- liver cirrhosis

- HCC longitudinal studies in NAFLD have shown that light-to-moderate alcohol use is associated with fibrosis progression and incident clinical liver disease, suggesting that there is no liversafe limit of alcohol intake in the presence of NAFLD; a J-shaped association between alcohol and all-cause mortality remains controversial. The interaction effects between alcohol and obesity make the present strict dichotomization of liver disease into alcoholic and NAFLD inappropriate, and require attention in future research, public health policy, individual counseling, and risk stratification.
\end{abstract}

Chronic liver disease (CLD) represents a growing health care problem. Globally, liver cirrhosis is the 11th leading cause of death, and hepatocellular carcinoma (HCC) is the 4th leading cause of cancer death. ${ }^{1}$ Mortality from liver diseases and HCC has been increasing in many countries. ${ }^{2,3}$ Cirrhosis is also one of the top 20 causes of disability-adjusted life years ${ }^{1}$ and represents a substantial economic burden.

Nonalcoholic fatty liver disease (NAFLD) and alcoholic liver disease (ALD) are the two main liver diseases worldwide. ${ }^{1}$ NAFLD, which is strongly interlinked with obesity and metabolic disorders, affects $25 \%$ of individuals in the population, increasing to more than $90 \%$ in the morbid obese. ${ }^{4}$ One study estimated that the annual direct medical costs from NAFLD alone are US $\$ 103$ billion in the United States and US $\$ 35$ billion in central Europe. ${ }^{5}$ ALD is the most common cause of liverrelated death in Western populations and a leading indication for liver transplantation in both Europe and the United States. ${ }^{1}$
NAFLD and ALD share many features in common, including genetic risk factors, many pathophysiological pathways, and histological features. ${ }^{6,7}$ Both NAFLD and ALD typically progress from steatosis to steatohepatitis, fibrosis, cirrhosis, and HCC. However, although most obese persons have NAFLD, less than $5 \%$ of NAFLD patients will ever develop complicated liver disease. ${ }^{8}$ Similarly, although there is a strong dose-response relationship between per capita alcohol consumption and liver cirrhosis mortality in the population, ${ }^{9}$ only 10 to $15 \%$ of heavy drinkers develop cirrhosis during their lifetime. ${ }^{10,11}$ Determinants of cirrhosis development remain incompletely understood.

Globally, overweight and obesity rates have nearly tripled since the 1970s. ${ }^{1}$ The current average prevalence of obesity in OECD (Organisation for Economic Co-operation and Development) countries is $23 \%$, with the United States having the highest rate (38\%). ${ }^{12}$ published online

February 18, 2020
Copyright $\odot 2020$ by Thieme Medical Publishers, Inc., 333 Seventh Avenue, New York, NY 10001, USA. Tel: +1(212) 760-0888.
DOI https://doi.org/ 10.1055/s-0040-1701443. ISSN 0272-8087. 
Alcohol consumption in the population varies in different regions, but around $60 \%$ of adults in Europe and the United States drink alcohol. Among the active drinkers (abstainers excluded), the average per capita annual consumption is 15 to $17 \mathrm{~L}$ of pure alcohol. ${ }^{13}$ Consequently, many persons with obesity and/or metabolic disorders also drink alcohol.

Recent studies emphasize the interaction between alcohol and obesity in the development of liver disease. ${ }^{14-16}$ Metabolic risk might sensitize the liver to alcohol damage and vice versa. Alcohol and obesity seems to have synergistic harm on the liver in certain situations ( - Fig. 1). ${ }^{14-17}$ We discuss recent evidence on interactions between alcohol and obesity, and their contribution to liver morbidity in the population.

\section{Effects of Alcohol on Obesity}

Pure ethanol contains $7 \mathrm{kcal} / \mathrm{g}$ and can significantly contribute to dietary calorific excess. Light-to-moderate alcohol intake does not seem to be associated with weight gain, whereas heavy drinking is. ${ }^{18,19}$ Light wine consumption might even protect against weight gain, ${ }^{19}$ but residual confounding from other associated lifestyle factors and dietary patterns cannot be excluded. Beer intake $>500 \mathrm{~mL} /$ day has been associated with abdominal obesity ("beer belly"). ${ }^{20}$

In contrast, persons with chronic alcohol abuse or cirrhosis tend to show increased energy expenditure with preferential use of lipids as fuel. This shift toward lipid oxidation to meet energy requirements is likely to contribute to the reduced overall fat mass in such persons. ${ }^{21}$

Alcohol has numerous complex effects on, for instance, food intake regulation, psychosocial well-being, sleeping, and depression symptoms, all of which may influence body weight. Such effects have substantial interindividual variation, and this may in part explain the somewhat conflicting results seen in studies $^{18,19}$ on the associations between alcohol and obesity.

\section{Obesity and Liver Disease}

Obesity is associated with NAFLD and is a cofactor in many other liver diseases. ${ }^{22-26}$ This cofactor effect of obesity may be medi- ated by the additive effect from coexistent NAFLD. Alternatively, obesity-associated metabolic abnormalities might exacerbate liver injury from other causes such as alcohol or viruses. ${ }^{27}$

Body mass index (BMI) has traditionally been the anthropometric measure of choice to diagnose overweight and obesity. However, alternative measures that reflect abdominal (also called visceral or central) adiposity, such as waist circumference and waist-hip ratio (WHR), seem superior to BMI in predicting incident liver disease. ${ }^{14,28-31}$

A recent population study that analyzed a large number of anthropometric measures in predicting incident severe liver disease found WHR to be the strongest predictor (men: hazard ratio [HR] for 1 standard deviation [SD] of 1.46 and c-statistics of 0.70; women: HR per 1 SD of 1.30 and c-statistics of 0.63$){ }^{30}$ This effect of WHR was independent of BMI, and BMI showed no predictive value independent of WHR. In this study, BMI was, in fact, the poorest predictor of all tested anthropometric measures (men: HR per 1 SD of 1.26 , c-statistics of 0.68 ; women: HR per 1 SD of 1.12 and c-statistics of 0.60 ). Recently, these findings have been confirmed by others. ${ }^{31,32}$ However, BMI seems to have a $\mathbf{U}$-shaped association with liver disease, ${ }^{22}$ and treating BMI as a linear predictor in many studies may have led to an attenuation of the risk effect of BMI.

The association between obesity and liver disease seems to be driven largely by metabolic risk, including insulin resistance, type 2 diabetes, dyslipidemia, and hypertension. ${ }^{33,34}$ A high waist circumference or WHR reflects the "appleshaped" central distribution of body fat, which is strongly associated with metabolic abnormalities.

In parallel, an increasing number of components of the metabolic syndrome (MetS), visceral obesity, type 2 diabetes, and insulin resistance are strongly associated with progression of NAFLD, ${ }^{35}$ whereas a high BMI per se is not directly associated with more advanced NAFLD stages. ${ }^{36,37}$ In fact, metabolically healthy ("pear-shaped") obesity was associated with less adipose tissue inflammation and less liver fibrosis than metabolically unhealthy ("apple-shaped") obesity. ${ }^{33}$

In a longitudinal population study, the metabolic features that best predicted incident advanced liver disease in nonrisk alcohol drinkers were abdominal obesity (HR: 1.03 per $\mathrm{cm}$

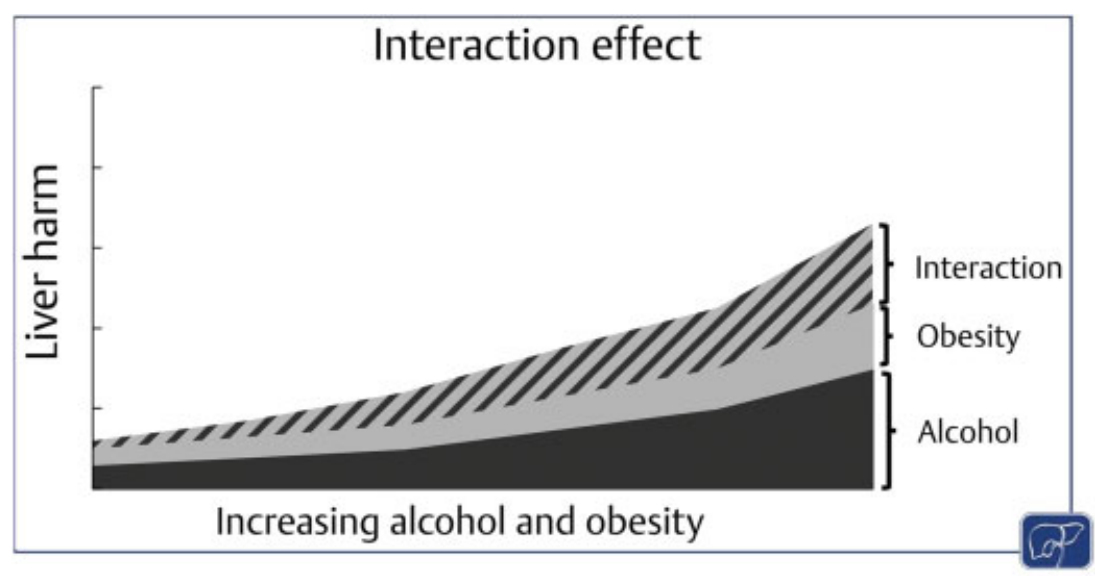

Fig. 1 The concept of synergistic interaction. Synergistic or supra-additive interaction refers to a situation in which the combined effect (liver toxicity) of two exposures (alcohol and obesity) is greater than the sum of their individual effects. 
increase in waist circumference), insulin resistance (HR: 1.04 per unit increase in homeostasis model of assessment of insulin resistance), and a low LDL (low-density lipoprotein) cholesterol level (HR: 0.54 per $\mathrm{mmol} / \mathrm{L}$ increase in LDL cholesterol). ${ }^{14}$ Therefore, measures of body fat distribution seems to have a predictive value for liver disease independent of other metabolic risk factors.

Features of the MetS also predict HCC and survival in CLD regardless of the primary etiology of the liver disease. ${ }^{38,39}$

\section{Alcoholic Liver Disease and Obesity}

The arbitrary threshold of alcohol intake currently used to discriminate ALD from NAFLD-30 g/day for men and $20 \mathrm{~g} /$ day for women-is based on the assumption that alcohol intake below these levels is insufficient to induce hepatic steatosis $^{40-42}$ and has a low risk of (alcoholic) cirrhosis. ${ }^{9}$ However, this threshold does not take into consideration the pattern of alcohol use, binge-drinking, beverage preference, or other demographic factors than gender.

Although in most cohort studies of "pure" alcoholic liver cirrhosis, ${ }^{43-45}$ patients have a history of consuming 6 to 12 drinks per day for an average of 30 to 40 years, these cohorts are preselected as typical ALD. Alcohol use at much lower levels may be a contributing factor in other types of liver disease labeled as nonalcoholic and thereby excluded from cohort studies of ALD. Alcohol toxicity seems to depend on the drinking pattern including frequency and quantity, beverage type (lower risk for wine), drinking outside meals, and binge-drinking. ${ }^{10,46-49}$ However, the drinking pattern alone is insufficient to explain liver disease development since it does not significantly differ from the pattern of individuals with longstanding alcohol-use disorder without liver disease. ${ }^{44,45}$ Alcoholic liver toxicity is also modified by genetics, gender, environmental factors, diet, gut microbiota, and comorbidity including obesity and metabolic risk. Changes in gut microbiota (dysbiosis) have been described in both ALD and NAFLD. ${ }^{50}$

The relevance of metabolic risk factors for liver disease progression may have been underestimated in cohort studies of ALD cases because many metabolic conditions such as obesity, dyslipidemia, and hypertension tend to disappear as end-stage liver disease develops. Indeed, among alcohol risk drinkers in the general population without manifest liver disease, abdominal obesity (HR: 1.6 per 1 SD increase in WHR), diabetes (HR: 3.4), hypertension (HR: 2.5), lipid abnormalities (HR: 2.1 for HDL cholesterol; HR: 1.2 for triglycerides), and inactive lifestyle (HR: 1.6 for exercise less than once monthly) predicted the development of advanced liver disease during follow-up independently of alcohol. ${ }^{51}$

NAFLD is known to recur in most patients after liver transplantation as transplantation does not treat the cause of steatosis. Interestingly, the rate of posttransplant steatosis in the ALD group was likewise particularly high (37 vs 26\%), despite alcohol abstinence, further indicating that many ALD patients also have underlying risk factors for NAFLD (metabolic risk, gut dysbiosis) contributing to their liver disease all along. ${ }^{52,53}$

\section{ALD and NAFLD: Common Pathophysiological Pathways}

There are considerable overlap and similarities in both the molecular pathways ${ }^{54}$ and liver histology between ALD and NAFLD. Both ALD and NAFLD are heterogenic diseases where multiple pathogenic mechanisms contribute to disease progression in any single patient. ${ }^{55,56}$ Common to both ALD and NAFLD is that transition from steatosis to steatohepatitis is related to mitochondrial dysfunction and oxidative stress. ${ }^{57}$ In addition, intestinal dysbiosis, barrier disruption, and endotoxemia are implicated in both ALD and NAFLD, ${ }^{55,58,59}$ and the diseases share a common genetic background. ${ }^{7}$ A single variant in the patatin-like phospholipase-containing domain 3 (PNPLA3) gene, the rs738409[G], has been associated with more rapid progression of fatty liver disease to fibrosis and cirrhosis, with an approximately two- to threefold risk for decompensated cirrhosis and HCC among carriers of the risk variant both in NAFLD and ALD. ${ }^{7,60-63}$ Other genetic risk variants, such as rs72613567:TA in HSD17B13, also overlap between NAFLD and ALD. 7,64

There are several mechanistic links between ALD and NAFLD. First, many animal models on alcoholic liver injury such as the basic Lieber-DeCarli model ${ }^{65}$ include a high-fat diet component, making them basically models of ALD-NAFLD interaction to begin with. Second, there seems to be a common activation of innate immune responses, with synergism between alcohol and obesity. ${ }^{66}$ Third, ethanol degradation through the cytochrome P450 2E1 (CYP2E1) pathway catalyzes hepatotoxic reactive oxygen species. ${ }^{59}$ Diabetes and obesity can induce CYP2E1, thereby causing relatively increased ethanol degradation through the CYP2E1 pathway, which might amplify alcohol hepatotoxicity. ${ }^{59}$ Fourth, according to the endogenous alcohol hypothesis, especially in obesity, the intestinal microbiome can produce ethanol to hepatotoxic levels. ${ }^{67}$ These findings have been substantiated by observations of upregulation of genes involved in alcohol metabolism pathways in NAFLD despite complete alcohol abstinence. ${ }^{67-69}$ Finally, alcohol can disrupt extrahepatic fat tissue function and cause adipocyte death with subsequent proinflammatory responses and increased lipolysis, thereby contributing to liver damage by indirect mechanisms. ${ }^{21}$ As an example of mechanistic synergism between alcohol and obesity, it has been shown that a high-fat diet sensitizes adipose tissue to alcohol-induced lipolysis. $^{21}$

In mice, moderate obesity (a $28-35 \%$ weight increase) and alcohol synergistically induce steatohepatitis and liver fibrosis over and beyond their individual effects. ${ }^{66}$ Possible mechanisms include increased plasma adiponectin activation, defective hepatic AMP (adenosine monophosphate)-activated protein kinase signaling, heightened endoplasmic reticulum stress, and suppression of genes involved in mitochondrial functions. ${ }^{66}$ Minato et al further showed that alcohol administered once a week in a binge-drinking fashion to mice with hepatic steatosis aggravated hepatic oxidative stress and promoted steatohepatitis from obesity-induced steatosis; no such effects of binge alcohol were seen in nonsteatotic mice. ${ }^{70}$ Duly et al similarly showed that on a background of high-fat 
diet-induced hepatic steatosis, intermittent binge-drinking synergistically increased steatosis, inflammation, and fibrosis in mice livers compared with binge-drinking or a high-fat diet alone. ${ }^{71}$ These findings suggest that binge-drinking particularly may serve as a "second hit" in the development of steatohepatitis in NAFLD.

\section{ALD and NAFLD: Epidemiological Interactions}

Several cross-sectional studies have shown combined effects of alcohol and obesity on transaminase levels ${ }^{72-74}$ and steatosis. ${ }^{17,75}$ In contrast, other studies have suggested protective effects of light alcohol intake in the form of a reduced prevalence of NAFLD and liver fibrosis, ${ }^{76-80}$ possibly driven by an association between light alcohol intake and improved lipid profiles, anti-inflammatory effects, and improved insulin sensitivity. ${ }^{81,82}$ As recently reviewed, ${ }^{83}$ however, there are major methodological concerns with these studies, including a cross-sectional design, use of surrogate end points, and incomplete adjustment for confounders such as physical activity, smoking, dietary factors (for instance, coffee), socioeconomic status, comorbidity, and ethnicity. Many studies failed to consider the pattern and type of alcohol use and the lifetime alcohol intake in addition to average alcohol intake, and did not separate between lifetime abstainers and current abstainers, the latter of which may be enriched in former heavy drinkers. In addition, underreporting of alcohol use is a major concern particularly when assessing patients who know they have a liver disease. In fact, recent studies analyzing specific alcohol-use markers such as ethyl glucuronide or phosphatidylethanol showed high rates (up to 57\%) of undetected moderate to high alcohol use in patients with presumed NAFLD. ${ }^{79,84}$ Prospective population cohort studies have an advantage over clinic-based studies in this regard since healthy participants are more likely to respond honestly.

A growing number of longitudinal population studies that excluded subjects with baseline liver disease report synergistic effects between alcohol use and obesity in the risk of future clinical liver disease, cirrhosis, and/or HCC. ${ }^{14,15,22,46,85}$ This interaction effect is most profound when obesity is measured by the $\mathrm{WHR}^{16}$ compared with BMI or waist circumference. ${ }^{14}$ Among men in the highest tertile of WHR, one daily alcohol drink yielded a similar relative risk for incident advanced liver disease as four daily drinks in nonobese men (-Fig. 2). ${ }^{16}$ This means that in the presence of marked central obesity, hepatic toxicity of alcohol increases by several folds.

Few studies have analyzed interactions for liver disease risk between alcohol and other metabolic factors besides obesity. In our general population study, we observed a marked interaction effect between alcohol risk use and diabetes in the development of incident liver disease ( - Fig. 3 ). ${ }^{14}$

In a recent U.S. population study of individuals with ultrasound-based hepatic steatosis, excessive alcohol use ( $>$ three drinks/day for men or $>1.5$ drinks/day for women) was associated with increased overall mortality (HR: 2.5) among persons with concomitant MetS but not among persons without MetS (HR: 1.1). ${ }^{86}$

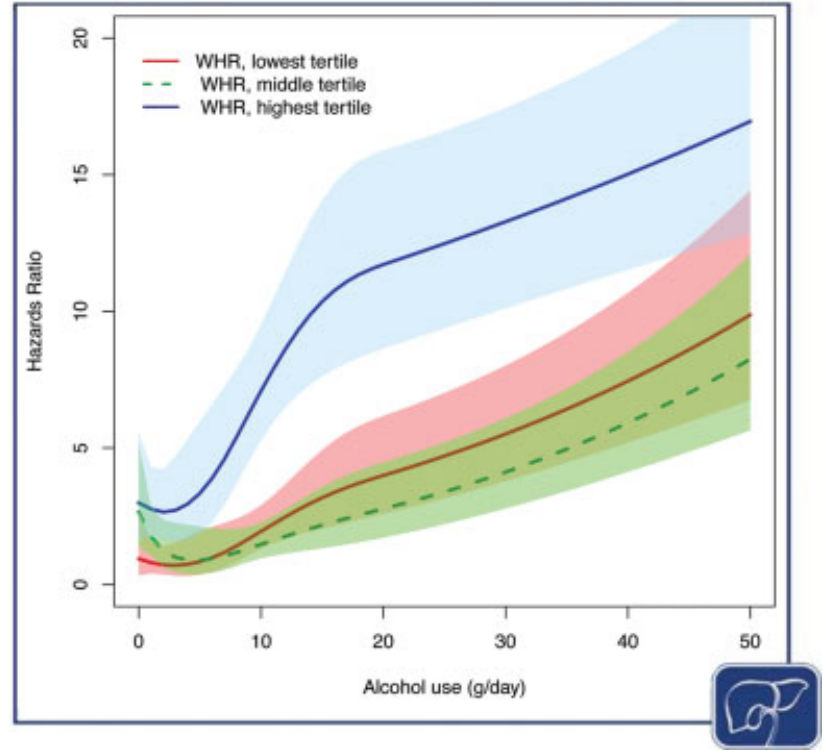

Fig. 2 Interaction between average alcohol intake (grams of ethanol per week) and WHR in the development of incident clinical liver disease among men in the Finnish general population. WHR, waist-hip ratio. (Reproduced with permission of Sahlman et al. ${ }^{16}$ )

\section{Binge-Drinking}

Intermittent heavy drinking or binge-drinking typically refers to drinking $60 \mathrm{~g}$ ethanol or more on one occasion at least once during the last month. ${ }^{13}$ Up to $26 \%$ of adults in Europe and 21\% in the United States report such binge-drinking at least once monthly. ${ }^{13}$ Unfortunately, epidemiological studies assessing alcohol use by a standard quantity-frequency approach tend to neglect the issue of binge-drinking. ${ }^{87}$

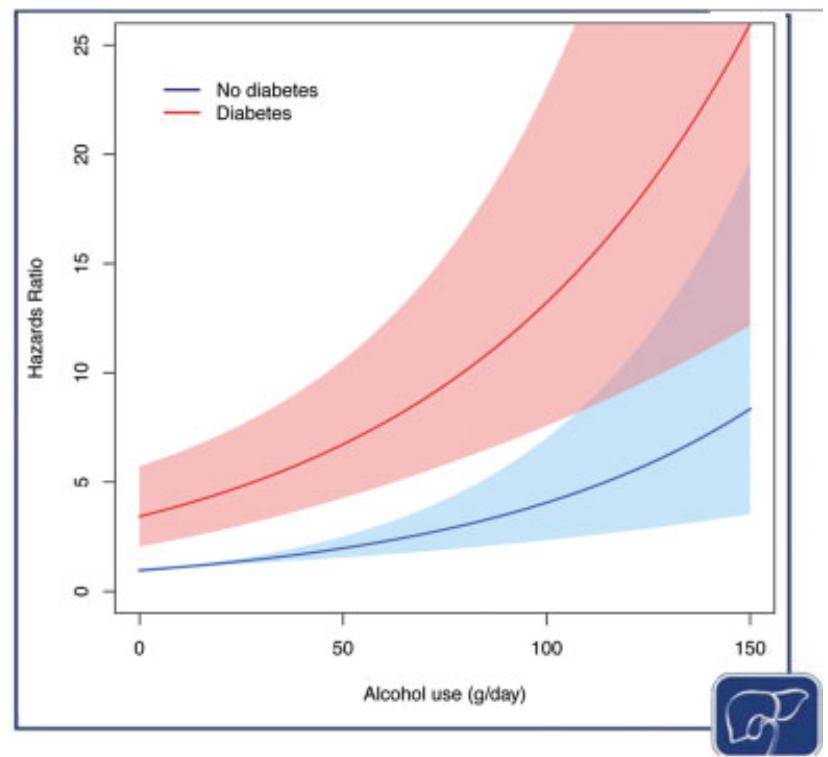

Fig. 3 Interaction between average alcohol intake (grams of ethanol per week) and diabetes in the development of incident clinical liver disease in the Finnish general population. (Reproduced with permission of Åberg et al. ${ }^{14}$ ) 
Alcoholic liver cirrhosis has typically been considered the consequence of daily drinking rather than intermittent binge-drinking, ${ }^{47}$ which has been explained by the liver's capacity to recover after each episode of drinking during the break from alcohol intake. However, in the context of obesity and NAFLD, the already injured steatotic liver may lack this capacity to recover. This hypothesis is supported by recent animal ${ }^{70,71}$ and human ${ }^{17}$ studies. Binge-drinking has several potentially harmful effects on the $\operatorname{liver}^{88}$ and was, for example, shown to induce insulin resistance that lasted even after blood alcohol levels had become undetectable. ${ }^{89}$

In a Finnish population study, we found that binge-drinking was associated with around threefold (HR: 2.8-3.5 for weekly binge-drinking depending on the level of adjustment) increased risk for advanced liver disease regardless of the level of average alcohol use. ${ }^{46}$ Moreover, we found an interaction effect between weekly binge-drinking and MetS. In fact, binge-drinking was a significant risk factor for liver disease only among those with the MetS but not among those without it (-Fig. 4). ${ }^{46}$

Another study of 71 patients with biopsy-proven NAFLD (alcohol intake $<140 \mathrm{~g} /$ week) found that binge-drinking (60 $\mathrm{g}$ ethanol per occasion for men, $48 \mathrm{~g}$ for women) as infrequently as once a month was associated with a more rapid progression of liver fibrosis. ${ }^{90}$

Furthermore, in recent population studies of NAFLD, binge-drinking emerged as an independent risk factor for progression to advanced clinical liver disease (HR: 1.5-2.7 among monthly to weekly binge drinkers) ${ }^{91}$ and all-cause death (HR: 1.5 among monthly binge drinkers). ${ }^{86}$ The corollary of these recent studies is that binge-drinking seems to be an important risk factor for advanced liver disease and mortality among persons with the MetS and/or hepatic steatosis.

\section{Are There Safe Limits of Alcohol Use in NAFLD?}

There are no randomized trials assessing light-to-moderate alcohol use in NAFLD. The existing literature comprises somewhat conflicting findings, as discussed earlier. A possible protective effect of alcohol from liver fibrosis has not been confirmed in longitudinal studies. ${ }^{82,90,92}$

One recent longitudinal study of 285 patients with biopsy-confirmed NAFLD found that alcohol intake of $\leq$ two drinks/day was associated with lower odds (odds ratio: 0.32 ) of histological steatohepatitis resolution compared with total abstinence. ${ }^{93}$ This finding is in sharp contrast to that in a previous cross-sectional study, in part, based on the same patients, which showed beneficial liver effects of low alcohol use. ${ }^{78} \mathrm{~A}$ recent Mendelian randomization study categorized patients with NAFLD based on a genotype in the aldehyde dehydrogenase gene that is associated with a lower level of alcohol consumption. ${ }^{94}$ The NAFLD patients genetically prone to drink less alcohol had less hepatic steatosis and less features of steatohepatitis on histology compared with the NAFLD patients lacking this genetic constraint to alcohol use. ${ }^{94}$ This points to the harmful effects of alcohol use in NAFLD.

In a recent population cohort study of 8,345 NAFLD patients (defined as a fatty liver index $>60$ ), we found no benefits from low alcohol intake with regard to the risk for incident advanced clinical liver disease. ${ }^{95}$ Advanced liver disease was defined as hospitalization, cancer, or death from liver cirrhosis, HCC, or equivalent clinically significant liver events. Consuming more than $10 \mathrm{~g} /$ day of alcohol increased the risk of advanced liver disease in a dosedependent fashion independent of relevant confounders. One drink per day of nonwine beverages or two drinks per day of wine doubled the risk for advanced liver disease.

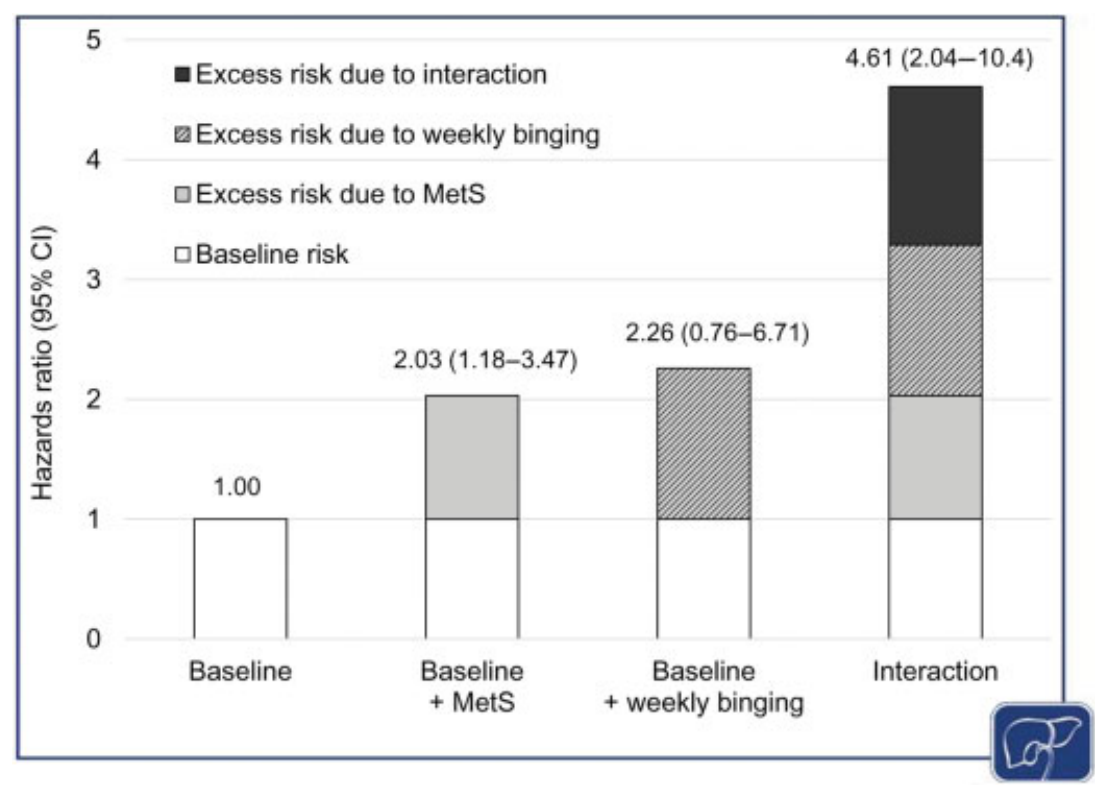

Fig. 4 Hazards ratios for the contribution of weekly binge-drinking and the MetS, and their combination (interaction) in the development of incident clinical liver disease in comparison with participants without MetS who reported no binge-drinking or binge-drinking less often than once monthly (baseline risk). Cl, confidence interval; MetS, metabolic syndrome. (Reproduced with permission of Åberg et al. ${ }^{46}$ ) 
Among risk drinkers, we found no influence of beverage type on the risk for liver disease. Similar findings were recently reported in a Korean study analyzing changes over time in noninvasive fibrosis markers. ${ }^{96}$

\section{Extrahepatic Outcomes}

In the general population, low alcohol intake has been associated with an increased risk of certain cancers ${ }^{92}$ but reduced risks of cardiovascular disease ${ }^{97}$; both of these associations were confirmed in our population NAFLD cohort. ${ }^{95}$ However, the association between alcohol and reduced cardiovascular risk may be limited by residual confounding. In fact, a recent study that prospectively assessed alcohol consumption over time found no association between alcohol use and the presence of cardiovascular risk factors or subclinical cardiovascular disease, ${ }^{98}$ although this study did not analyze clinical cardiovascular events. A recent analysis of individual participant data of 599,912 current drinkers in 83 prospective studies assessing risk thresholds for alcohol consumption ${ }^{99}$ concluded that the threshold for lowest risk of all-cause mortality was $\leq 100 \mathrm{~g}$ ethanol per week. For cardiovascular disease subtypes other than myocardial infarction, the alcohol intake level with the lowest risk was zero.

There are conflicting results regarding the association between low alcohol intake and all-cause mortality in subjects with NAFLD. ${ }^{46,86,95}$ While we found a J-shaped association in our Finnish study, ${ }^{95}$ two recent U.S. studies reported contradictory results. ${ }^{46,86}$ Hajifathalian et al ${ }^{100}$ reported a Jshaped association between drinking and all-cause mortality, whereas Younossi et $\mathrm{al}^{86}$ found no reduced mortality among light drinkers. Although both studies were based on the National Health and Nutrition Examination Survey data, they differed in their definition of hepatic steatosis (ultrasound vs. hepatic steatosis index) and the variables adjusted for in multivariate analyses. ${ }^{46,86}$

In patients with NAFLD cirrhosis, low alcohol use ( $<30 \mathrm{~g} /$ day for men and $<20 \mathrm{~g} /$ day for women) compared with abstinence was associated with an increased risk of death or liver transplantation (HR: 2.3), hepatic decompensation (HR: 1.7), and HCC (HR: 3.2), thereby reinforcing the need for absolute alcohol abstinence in patients with liver cirrhosis. ${ }^{101}$

\section{Implications of ALD/NAFLD Interactions}

Alcohol, obesity, and metabolic disorders are continuous variables as such, not dichotomic, with additive harmful interactions. Given the numerous similarities, overlapping features, and interactions both mechanistically and clinically, the current dichotomization of ALD and NAFLD into mutually exclusive diseases seems inappropriate.

In the struggle to tackle a growing burden of liver disease in the population, the dichotomized view of ALD and NAFLD is most likely a suboptimal strategy for the identification and prognostication of at-risk individuals in the general population and in counselling regarding risks. Indeed, in a large number of CLD cases in the population, the liver disease seems to be driven by joint effects of alcohol and metabolic risk. ${ }^{102}$
There seems to be a need for a more holistic approach, where all the risk factors are considered at the same time as a continuum. In addition to measuring liver fibrosis stage, there is a need for indices that quantify a person's individual risk for developing clinical liver disease. Such indices should ideally incorporate key pathophysiological drivers of liver disease, making it also usable in assessing response to therapeutic interventions. Suggestions for future research are summarized in - Table $\mathbf{1}$.

In clinical practice, alcohol consumption patterns beyond average alcohol intake should be assessed in all patients with liver disease and among those with obesity or the MetS. Longitudinal population studies suggest that there is no general liver-safe limit of alcohol use in the presence of metabolic risk factors or NAFLD. Although low alcohol use may improve insulin sensitivity and thereby possibly reduce hepatic fat content, the numerous detrimental effects of alcohol extend beyond simple steatosis. There is currently no clinical evidence to support that low alcohol would protect from symptomatic liver disease. In addition, in any given individual, the level at which alcohol becomes harmful may vary depending on genetics, intake pattern, beverage type, lifetime consumption, and comorbidity. Because of this individual variation, which is difficult to define, no general safe limit of alcohol can be determined. Even low alcohol intake is associated with cancer risk. In advanced liver fibrosis or particular risk for progressive liver disease, total alcohol abstinence is advisable. On the other hand, among active drinkers with liver disease, attempts are needed to

Table 1 Suggestions for future research on ALD/NAFLD interactions

\begin{tabular}{|l|}
\hline $\begin{array}{l}\text { Large longitudinal studies, with alcohol consumption } \\
\text { assessed repeatedly over time }\end{array}$ \\
\hline $\begin{array}{l}\text { Quantification of alcohol consumption by objective } \\
\text { biomarkers such as phosphatidylethanol }\end{array}$ \\
\hline $\begin{array}{l}\text { Factors influencing individual susceptibility to alcoholic liver } \\
\text { toxicity, including genetics }\end{array}$ \\
\hline The effects of binge-drinking on metabolic fatty liver disease \\
\hline $\begin{array}{l}\text { Mechanisms of interaction between alcohol and metabolic } \\
\text { abnormalities on liver toxicity }\end{array}$ \\
\hline $\begin{array}{l}\text { Studies on which specific metabolic factors drive the harmful } \\
\text { interaction effects with alcohol for liver disease }\end{array}$ \\
\hline $\begin{array}{l}\text { Interactions between genetic risk, alcohol use, and } \\
\text { metabolic abnormalities in the development of liver disease }\end{array}$ \\
\hline $\begin{array}{l}\text { Studies on how to incorporate the interaction effects } \\
\text { between alcohol and metabolic factors in risk stratification }\end{array}$ \\
\hline $\begin{array}{l}\text { Studies on how to incorporate the interaction effects } \\
\text { between alcohol and metabolic factors in the diagnostic } \\
\text { criteria of fatty liver disease }\end{array}$ \\
\hline $\begin{array}{l}\text { Studies on how to incorporate the interaction effects } \\
\text { between alcohol and metabolic factors in therapeutic } \\
\text { interventions for fatty liver disease }\end{array}$ \\
\hline
\end{tabular}

Abbreviations: ALD, alcoholic liver disease; NAFLD, nonalcoholic fatty liver disease. 
also address metabolic and dietary factors, in addition to alcohol withdrawal. ${ }^{103}$

\section{Conclusion}

There are strong epidemiological and experimental evidences that alcohol and metabolically unhealthy obesity exerts synergistic effects on the progression toward liver cirrhosis. In fact, a large proportion of the population who develop liver disease have a combination of NAFLD and ALD risk factors. Recent longitudinal studies suggest that even low alcohol use increases the risk of advanced clinical liver disease in NAFLD. On the other hand, several metabolic factors increase the risk of cirrhosis among alcohol risk drinkers. At a population level, the combined effects of alcohol and metabolic factors may be particularly relevant in the large number of persons who are overweight or obese and consume just a little too much alcohol.

\section{Conflict of Interest}

None.

\section{References}

1 Asrani SK, Devarbhavi H, Eaton J, Kamath PS. Burden of liver diseases in the world. J Hepatol 2019;70(01):151-171

2 Pimpin L, Cortez-Pinto H, Negro F, et al; EASL HEPAHEALTH Steering Committee. Burden of liver disease in Europe: epidemiology and analysis of risk factors to identify prevention policies. J Hepatol 2018;69(03):718-735

3 Kim Y, Ejaz A, Tayal A, et al. Temporal trends in population-based death rates associated with chronic liver disease and liver cancer in the United States over the last 30 years. Cancer 2014;120(19): 3058-3065

4 Younossi ZM, Koenig AB, Abdelatif D, Fazel Y, Henry L, Wymer M. Global epidemiology of nonalcoholic fatty liver disease-metaanalytic assessment of prevalence, incidence, and outcomes. Hepatology 2016;64(01):73-84

5 Younossi ZM, Blissett D, Blissett R, et al. The economic and clinical burden of nonalcoholic fatty liver disease in the United States and Europe. Hepatology 2016;64(05):1577-1586

6 Boyle M, Masson S, Anstee QM. The bidirectional impacts of alcohol consumption and the metabolic syndrome: cofactors for progressive fatty liver disease. J Hepatol 2018;68(02): 251-267

7 Anstee QM, Seth D, Day CP. Genetic factors that affect risk of alcoholic and nonalcoholic fatty liver disease. Gastroenterology 2016;150(08):1728-1744.e7

8 Rinella M, Charlton M. The globalization of nonalcoholic fatty liver disease: prevalence and impact on world health. Hepatology 2016;64(01):19-22

9 Rehm J, Taylor B, Mohapatra S, et al. Alcohol as a risk factor for liver cirrhosis: a systematic review and meta-analysis. Drug Alcohol Rev 2010;29(04):437-445

10 Bellentani S, Saccoccio G, Costa G, et al; The Dionysos Study Group. Drinking habits as cofactors of risk for alcohol induced liver damage. Gut 1997;41(06):845-850

11 Teli MR, Day CP, Burt AD, Bennett MK, James OF. Determinants of progression to cirrhosis or fibrosis in pure alcoholic fatty liver. Lancet 1995;346(8981):987-990

12 OECD. Obesity Update. Available at: https://www.oecd.org/els/ health-systems/Obesity-Update-2017.pdf. Accessed August 1,2019

13 World Health Organization. Global status report on alcohol and health 2018. Available at: https://www.who.int/substance_abuse/ publications/global_alcohol_report/gsr_2018/en/. Accessed August 1,2019

14 Åberg F, Helenius-Hietala J, Puukka P, Färkkilä M, Jula A. Interaction between alcohol consumption and metabolic syndrome in predicting severe liver disease in the general population. Hepatology 2018;67(06):2141-2149

15 Hart CL, Morrison DS, Batty GD, Mitchell RJ, Davey Smith G. Effect of body mass index and alcohol consumption on liver disease: analysis of data from two prospective cohort studies. BMJ 2010; 340:c1240

16 Sahlman P. Nissinen M, Puukka P, et al. Genetic and lifestyle risk factors for advanced liver disease among men and women. J Gastroenterol Hepatol 2019 (e-pub ahead of print) . Doi: 10.1111/jgh. 14770

17 Lau K, Baumeister SE, Lieb W, et al. The combined effects of alcohol consumption and body mass index on hepatic steatosis in a general population sample of European men and women. Aliment Pharmacol Ther 2015;41(05):467-476

18 Traversy G, Chaput JP. Alcohol consumption and obesity: an update. Curr Obes Rep 2015;4(01):122-130

19 Sayon-Orea C, Martinez-Gonzalez MA, Bes-Rastrollo M. Alcohol consumption and body weight: a systematic review. Nutr Rev 2011;69(08):419-431

20 Bendsen NT, Christensen R, Bartels EM, et al. Is beer consumption related to measures of abdominal and general obesity? A systematic review and meta-analysis. Nutr Rev 2013;71(02):67-87

21 Parker R, Kim SJ, Gao B. Alcohol, adipose tissue and liver disease: mechanistic links and clinical considerations. Nat Rev Gastroenterol Hepatol 2018;15(01):50-59

22 Liu B, Balkwill A, Reeves G, Beral V; Million Women Study Collaborators. Body mass index and risk of liver cirrhosis in middle aged UK women: prospective study. BMJ 2010;340:c912

23 Hagström H, Tynelius P, Rasmussen F. High BMI in late adolescence predicts future severe liver disease and hepatocellular carcinoma: a national, population-based cohort study in 1.2 million men. Gut 2018;67(08):1536-1542

24 Harris R, Harman DJ, Card TR, Aithal GP, Guha IN. Prevalence of clinically significant liver disease within the general population, as defined by non-invasive markers of liver fibrosis: a systematic review. Lancet Gastroenterol Hepatol 2017;2(04):288-297

25 Diehl AM, Day C. Cause, pathogenesis, and treatment of nonalcoholic steatohepatitis. N Engl J Med 2017;377(21):2063-2072

26 Caballería L, Pera G, Arteaga I, et al. High prevalence of liver fibrosis among european adults with unknown liver disease: a population-based study. Clin Gastroenterol Hepatol 2018;16 (07):1138-1145.e5

27 Chiang DJ, McCullough AJ. The impact of obesity and metabolic syndrome on alcoholic liver disease. Clin Liver Dis 2014;18(01): 157-163

28 Pang Q, Zhang JY, Song SD, et al. Central obesity and nonalcoholic fatty liver disease risk after adjusting for body mass index. World J Gastroenterol 2015;21(05):1650-1662

29 Ioannou GN, Weiss NS, Boyko EJ, et al. Is central obesity associated with cirrhosis-related death or hospitalization? A population-based, cohort study. Clin Gastroenterol Hepatol 2005;3 (01):67-74

30 Andreasson A, Carlsson AC, Önnerhag K, Hagström H. Waist/hip ratio better predicts development of severe liver disease within 20 years than body mass index: a population-based cohort study. Clin Gastroenterol Hepatol 2017;15(08):1294-1301.e2

31 Åberg F, Jula A. The sagittal abdominal diameter: role in predicting severe liver disease in the general population. Obes Res Clin Pract 2018;12(04):394-396

32 Schult A, Mehlig K, Björkelund C, Wallerstedt S, Kaczynski J. Waistto-hip ratio but not body mass index predicts liver cirrhosis in women. Scand J Gastroenterol 2018;53(02):212-217

33 Gutiérrez-Grobe Y, Juárez-Hernández E, Sánchez-Jiménez BA, et al. Less liver fibrosis in metabolically healthy compared with 
metabolically unhealthy obese patients with non-alcoholic fatty liver disease. Diabetes Metab 2017;43(04):332-337

34 Ampuero J, Aller R, Gallego-Durán R, et al; HEPAmet Registry. The effects of metabolic status on non-alcoholic fatty liver diseaserelated outcomes, beyond the presence of obesity. Aliment Pharmacol Ther 2018;48(11-12):1260-1270

35 Younossi ZM. Non-alcoholic fatty liver disease - a global public health perspective. J Hepatol 2019;70(03):531-544

36 Singh S, Allen AM, Wang Z, Prokop LJ, Murad MH, Loomba R. Fibrosis progression in nonalcoholic fatty liver vs nonalcoholic steatohepatitis: a systematic review and meta-analysis of paired-biopsy studies. Clin Gastroenterol Hepatol 2015;13 (04):643-54.e1, 9, quiz e39-e40

37 Argo CK, Northup PG, Al-Osaimi AM, Caldwell SH. Systematic review of risk factors for fibrosis progression in non-alcoholic steatohepatitis. J Hepatol 2009;51(02):371-379

38 Wainwright P, Scorletti E, Byrne CD. Type 2 diabetes and hepatocellular carcinoma: risk factors and pathogenesis. Curr Diab Rep 2017;17(04):20-017

39 Stepanova M, Rafiq N, Younossi ZM. Components of metabolic syndrome are independent predictors of mortality in patients with chronic liver disease: a population-based study. Gut 2010; 59(10):1410-1415

40 Kechagias S, Zanjani S, Gjellan S, et al. Effects of moderate red wine consumption on liver fat and blood lipids: a prospective randomized study. Ann Med 2011;43(07):545-554

41 European Association for the Study of the Liver (EASL); European Association for the Study of Diabetes (EASD); European Association for the Study of Obesity (EASO). EASL-EASD-EASO Clinical Practice Guidelines for the management of non-alcoholic fatty liver disease. J Hepatol 2016;64(06):1388-1402

42 Chalasani N, Younossi Z, Lavine JE, et al. The diagnosis and management of nonalcoholic fatty liver disease: practice guidance from the American Association for the Study of Liver Diseases. Hepatology 2018;67(01):328-357

43 Hatton J, Burton A, Nash H, Munn E, Burgoyne L, Sheron N. Drinking patterns, dependency and life-time drinking history in alcohol-related liver disease. Addiction 2009;104(04):587592

44 Stokkeland K, Hilm G, Spak F, Franck J, Hultcrantz R. Different drinking patterns for women and men with alcohol dependence with and without alcoholic cirrhosis. Alcohol Alcohol 2008;43(01): 39-45

45 Nielsen JK, Olafsson S, Bergmann OM, et al. Lifetime drinking history in patients with alcoholic liver disease and patients with alcohol use disorder without liver disease. Scand J Gastroenterol 2017;52(6-7):762-767

46 Åberg F, Helenius-Hietala J, Puukka P, Jula A. Binge drinking and the risk of liver events: a population-based cohort study. Liver Int 2017;37(09):1373-1381

47 Simpson RF, Hermon C, Liu B, et al; Million Women Study Collaborators. Alcohol drinking patterns and liver cirrhosis risk: analysis of the prospective UK Million Women Study. Lancet Public Health 2019;4(01):e41-e48

48 Askgaard G, Grønbæk M, Kjær MS, Tjønneland A, Tolstrup JS. Alcohol drinking pattern and risk of alcoholic liver cirrhosis: a prospective cohort study. J Hepatol 2015;62(05):1061-1067

49 Naveau S, Giraud V, Borotto E, Aubert A, Capron F, Chaput JC. Excess weight risk factor for alcoholic liver disease. Hepatology 1997;25(01):108-111

50 Betrapally NS, Gillevet PM, Bajaj JS. Changes in the intestinal microbiome and alcoholic and nonalcoholic liver diseases: causes or effects? Gastroenterology 2016;150(08):1745-1755.e3

51 Åberg F, Puukka P, Sahlman P, et al. Metabolic risk factors for advanced liver disease among alcohol risk users in the general population. J Hepatol 2019;70(Suppl 1):E273

52 Losurdo G, Castellaneta A, Rendina M, Carparelli S, Leandro G, Di Leo A. Systematic review with meta-analysis: de novo non- alcoholic fatty liver disease in liver-transplanted patients. Aliment Pharmacol Ther 2018;47(06):704-714

53 Hejlova I, Honsova E, Sticova E, et al. Prevalence and risk factors of steatosis after liver transplantation and patient outcomes. Liver Transpl 2016;22(05):644-655

54 Sookoian S, Pirola CJ. Systems biology elucidates common pathogenic mechanisms between nonalcoholic and alcoholic-fatty liver disease. PLoS One 2013;8(03):e58895

55 Nagy LE, Ding WX, Cresci G, Saikia P, Shah VH. Linking pathogenic mechanisms of alcoholic liver disease with clinical phenotypes. Gastroenterology 2016;150(08):1756-1768

56 Machado MV, Diehl AM. Pathogenesis of nonalcoholic steatohepatitis. Gastroenterology 2016;150(08):1769-1777

57 Sakaguchi S, Takahashi S, Sasaki T, Kumagai T, Nagata K. Progression of alcoholic and non-alcoholic steatohepatitis: common metabolic aspects of innate immune system and oxidative stress. Drug Metab Pharmacokinet 2011;26(01):30-46

58 Bajaj JS. Alcohol, liver disease and the gut microbiota. Nat Rev Gastroenterol Hepatol 2019;16(04):235-246

59 Nagata K, Suzuki H, Sakaguchi S. Common pathogenic mechanism in development progression of liver injury caused by non-alcoholic or alcoholic steatohepatitis. J Toxicol Sci 2007;32(05):453-468

60 Stickel F, Moreno C, Hampe J, Morgan MY. The genetics of alcohol dependence and alcohol-related liver disease. J Hepatol 2017;66 (01):195-211

61 Dongiovanni P, Donati B, Fares R, et al. PNPLA3 I148M polymorphism and progressive liver disease. World J Gastroenterol 2013; 19(41):6969-6978

62 Falleti E, Cussigh A, Cmet S, Fabris C, Toniutto P. PNPLA3 rs738409 and TM6SF2 rs58542926 variants increase the risk of hepatocellular carcinoma in alcoholic cirrhosis. Dig Liver Dis 2016;48(01):69-75

63 Grimaudo S, Pipitone RM, Pennisi G, et al. Association between PNPLA3 rs738409 C>G variant and liver-related outcomes in patients with non-alcoholic fatty liver disease. Clin Gastroenterol Hepatol 2019:S1542-3565(19)30886-9

64 Abul-Husn NS, Cheng X, Li AH, et al. A protein-truncating HSD17B13 variant and protection from chronic liver disease. N Engl J Med 2018;378(12):1096-1106

65 Guo F, Zheng K, Benedé-Ubieto R, Cubero FJ, Nevzorova YA. The Lieber-DeCarli diet-a flagship model for experimental alcoholic liver disease. Alcohol Clin Exp Res 2018;42(10):1828-1840

66 Xu J, Lai KKY, Verlinsky A, et al. Synergistic steatohepatitis by moderate obesity and alcohol in mice despite increased adiponectin and p-AMPK. J Hepatol 2011;55(03):673-682

67 de Medeiros IC, de Lima JG. Is nonalcoholic fatty liver disease an endogenous alcoholic fatty liver disease? - a mechanistic hypothesis. Med Hypotheses 2015;85(02):148-152

68 Cope K, Risby T, Diehl AM. Increased gastrointestinal ethanol production in obese mice: implications for fatty liver disease pathogenesis. Gastroenterology 2000;119(05):1340-1347

69 Baker SS, Baker RD, Liu W, Nowak NJ, Zhu L. Role of alcohol metabolism in non-alcoholic steatohepatitis. PLoS One 2010;5 (03):e9570

70 Minato T, Tsutsumi M, Tsuchishima M, et al. Binge alcohol consumption aggravates oxidative stress and promotes pathogenesis of NASH from obesity-induced simple steatosis. Mol Med 2014;20:490-502

71 Duly AM, Alani B, Huang EY, et al. Effect of multiple binge alcohol on diet-induced liver injury in a mouse model of obesity. Nutr Diabetes 2015;5:e154

72 Ruhl CE, Everhart JE. Joint effects of body weight and alcohol on elevated serum alanine aminotransferase in the United States population. Clin Gastroenterol Hepatol 2005;3(12):1260-1268

73 Alatalo PI, Koivisto HM, Hietala JP, Puukka KS, Bloigu R, Niemelä OJ. Effect of moderate alcohol consumption on liver enzymes increases with increasing body mass index. Am J Clin Nutr 2008; 88(04):1097-1103 
74 Loomba R, Bettencourt R, Barrett-Connor E. Synergistic association between alcohol intake and body mass index with serum alanine and aspartate aminotransferase levels in older adults: the Rancho Bernardo Study. Aliment Pharmacol Ther 2009;30 (11-12):1137-1149

75 Bellentani S, Saccoccio G, Masutti F, et al. Prevalence of and risk factors for hepatic steatosis in Northern Italy. Ann Intern Med 2000;132(02):112-117

76 Sookoian S, Castaño GO, Pirola CJ. Modest alcohol consumption decreases the risk of non-alcoholic fatty liver disease: a metaanalysis of 43175 individuals. Gut 2014;63(03):530-532

77 Moriya A, Iwasaki Y, Ohguchi S, et al. Alcohol consumption appears to protect against non-alcoholic fatty liver disease. Aliment Pharmacol Ther 2011;33(03):378-388

78 Dunn W, Sanyal AJ, Brunt EM, et al. Modest alcohol consumption is associated with decreased prevalence of steatohepatitis in patients with non-alcoholic fatty liver disease (NAFLD).J Hepatol 2012;57(02):384-391

79 Hagström H, Nasr P, Ekstedt M, et al. Low to moderate lifetime alcohol consumption is associated with less advanced stages of fibrosis in non-alcoholic fatty liver disease. Scand J Gastroenterol 2017;52(02):159-165

80 Kwon HK, Greenson JK, Conjeevaram HS. Effect of lifetime alcohol consumption on the histological severity of non-alcoholic fatty liver disease. Liver Int 2014;34(01):129-135

81 Roerecke M, Rehm J. Alcohol consumption, drinking patterns, and ischemic heart disease: a narrative review of meta-analyses and a systematic review and meta-analysis of the impact of heavy drinking occasions on risk for moderate drinkers. BMC Med 2014;12:182-014

82 Pietraszek A, Gregersen S, Hermansen K. Alcohol and type 2 diabetes. A review. Nutr Metab Cardiovasc Dis 2010;20(05):366-375

83 Ajmera VH, Terrault NA, Harrison SA. Is moderate alcohol use in nonalcoholic fatty liver disease good or bad? A critical review. Hepatology 2017;65(06):2090-2099

84 Staufer K, Strebinger G, Huber-Schoenauer U, et al. Ethyl glucuronide in hair uncovers a high rate of harmful alcohol consumption in patients with presumed NAFLD. J Hepatol 2019;70 (Suppl 1):E784

85 Loomba R, Yang HI, Su J, et al. Synergism between obesity and alcohol in increasing the risk of hepatocellular carcinoma: a prospective cohort study. Am J Epidemiol 2013;177(04):333-342

86 Younossi ZM, Stepanova M, Ong J, et al; Global NASH Council. Effects of alcohol consumption and metabolic syndrome on mortality in patients with nonalcoholic and alcohol-related fatty liver disease. Clin Gastroenterol Hepatol 2019;17(08):1625-1633.e1

87 Stahre M, Naimi T, Brewer R, Holt J. Measuring average alcohol consumption: the impact of including binge drinks in quantityfrequency calculations. Addiction 2006;101(12):1711-1718

88 Llerena S, Arias-Loste MT, Puente A, Cabezas J, Crespo J, Fábrega E. Binge drinking: burden of liver disease and beyond. World J Hepatol 2015;7(27):2703-2715

89 Lindtner C, Scherer T, Zielinski E, et al. Binge drinking induces whole-body insulin resistance by impairing hypothalamic insulin action. Sci Transl Med 2013;5(170):170ra14
90 Ekstedt M, Franzén LE, Holmqvist M, et al. Alcohol consumption is associated with progression of hepatic fibrosis in non-alcoholic fatty liver disease. Scand J Gastroenterol 2009;44(03):366-374

91 Åberg F, Puukka P, Sahlman P, et al. In NAFLD, alcohol drinking habits and genetics predict progression to advanced liver disease: follow-up of population surveys. J Hepatol 2019;70 (Suppl 1):E141

92 Cao Y, Willett WC, Rimm EB, Stampfer MJ, Giovannucci EL. Light to moderate intake of alcohol, drinking patterns, and risk of cancer: results from two prospective US cohort studies. BMJ 2015;351:h4238

93 Ajmera V, Belt P, Wilson LA, et al; Nonalcoholic Steatohepatitis Clinical Research Network. Among patients with nonalcoholic fatty liver disease, modest alcohol use is associated with less improvement in histologic steatosis and steatohepatitis. Clin Gastroenterol Hepatol 2018;16(09):1511-1520.e5

94 Sookoian S, Flichman D, Castaño GO, Pirola CJ. Mendelian randomisation suggests no beneficial effect of moderate alcohol consumption on the severity of nonalcoholic fatty liver disease. Aliment Pharmacol Ther 2016;44(11-12):1224-1234

95 Åberg F, Puukka P, Salomaa V, et al. Risks of light and moderate alcohol use in fatty liver disease: follow-up of population cohorts. Hepatology 2019 (e-pub ahead of print). Doi: 10.1002/hep.30864

96 Chang Y, Cho YK, Kim Y, et al. Nonheavy drinking and worsening of noninvasive fibrosis markers in nonalcoholic fatty liver disease: a cohort study. Hepatology 2019;69(01):64-75

97 Di Castelnuovo A, Costanzo S, Bagnardi V, Donati MB, Iacoviello L, de Gaetano G. Alcohol dosing and total mortality in men and women: an updated meta-analysis of 34 prospective studies. Arch Intern Med 2006;166(22):2437-2445

98 VanWagner LB, Ning H, Allen NB, et al. Alcohol use and cardiovascular disease risk in patients with nonalcoholic fatty liver disease. Gastroenterology 2017;153(05):1260-1272.e3

99 Wood AM, Kaptoge S, Butterworth AS, et al; Emerging Risk Factors Collaboration/EPIC-CVD/UK Biobank Alcohol Study Group. Risk thresholds for alcohol consumption: combined analysis of individual-participant data for 599912 current drinkers in 83 prospective studies. Lancet 2018;391 (10129):1513-1523

100 Hajifathalian K, Torabi Sagvand B, McCullough AJ. Effect of alcohol consumption on survival in non-alcoholic fatty liver disease: A national prospective cohort study. Hepatology 2019;70(02):511-521

101 Vilar-Gomez E, Calzadilla-Bertot L, Wai-Sun Wong V, et al. Fibrosis severity as a determinant of cause-specific mortality in patients with advanced nonalcoholic fatty liver disease: a multi-national cohort study. Gastroenterology 2018;155(02):443-457.e17

102 Åberg F, Puukka P, Salomaa V, et al. Combined effects of alcohol and metabolic disorders in patients with chronic liver disease. Clin Gastroenterol Hepatol 2019:S1542-3565(19)30727-X

103 Irvine L, Crombie IK, Cunningham KB, et al. Modifying alcohol consumption to reduce obesity: a randomized controlled feasibility study of a complex community-based intervention for men. Alcohol Alcohol 2017;52(06):677-684 